Article

\title{
Response of Malaria Hazard and Risk to a Hydroelectric Dam in Ethiopia using GIS and remote sensing
}

\author{
Abigail Smith ${ }^{1}$ and Sunhui Sim ${ }^{2}$ * \\ 1 University of North Alabama, 1 Harrison Plaza Florence, AL 35632 USA; asmith38@una.edu \\ 2 University of North Alabama, 1 Harrison Plaza Florence, AL 35632 USA; ssim@una.edu \\ * Correspondence: ssim@una.edu; Tel.:+1256-765-4250
}

\begin{abstract}
Malaria is a disease spread by female mosquitos of the Anopheles genus. It is acutely prevalent in Sub-Saharan Africa, where $90 \%$ of malaria deaths occur annually. One Sub-Saharan African country historically impacted by malaria is Ethiopia. In the past twenty years, malaria prevalence has decreased throughout Sub-Saharan Africa; yet, anthropogenic environmental changes are changing the landscape of malaria. Scholarly literature has cited a positive relationship between hydroelectric dams and malaria in Sub-Saharan Africa. Ethiopia is currently expanding their hydroelectric infrastructure. The Gilgel Gibe III Dam is located on the Omo River in Southwestern Ethiopia. It began generating electricity in 2015 and its reservoir has a capacity of 14,700 million $\mathrm{m} 3$ of water. This research utilized Geographic Information Systems and Remote Sensing to identify populations at an increased risk of malaria due to Gilgel Gibe III Dam. Two different techniques were employed: the proximity approach and the remote sensing approach. The proximity approach was based on distance from the reservoir. It identified all populations living within three kilometers of the reservoir as being at an increased risk. The remote sensing approach evaluated the slope, elevation, water content, and land surface temperature of the study area to create a mosquito breeding habitat risk map. Then, populations living within three kilometers of the two main High-Risk areas were identified. This study suggests that mosquito breeding habitat risk is not equally distributed throughout the Gilgel Gibe III Reservoir. This causes certain populations to be at a heightened risk of malaria.
\end{abstract}

Keywords: Malaria; Risk Maps; Remote Sensing, Ethiopia, Hydroelectric Dams

\section{Introduction}

Malaria is a parasitic infection that is spread by female mosquitos of the Anopheles genus. Its rate of transmission is highly dependent upon environmental conditions. Temperature is a key component because successful transmission of malaria parasites requires warm environments (Centers for Disease Control and Prevention, 2018). Malaria causes illness and death in tropical and sub-tropical countries throughout the world (Yewhalew et al., 2009). It is acutely prevalent in SubSaharan Africa where $90 \%$ of all malaria deaths occur killing approximately 400,000 people each year (Centers for Disease Control and Prevention, 2018). There are four malaria parasites of the Plasmodium genus that cause malaria in humans. (Hay et al., 2004). The most fatal malaria parasite in Sub-Saharan Africa is Plasmodium falciparum (Centers for Disease Control and Prevention, 2018).

One Sub-Saharan African country historically impacted by malaria is Ethiopia (Ayele et al., 2012). Two types of malaria parasites, Plasmodium falciparum and Plasmodium vivax, are abundant in the country. Malaria transmission in Ethiopia occurs cyclically increasing in September to November after the rainy season, June to August (Ghebryesus et al., 1999).

In 2005, health organizations, led by the Roll Back Malaria Initiative sought to halve the global malaria burden by 2010. They implemented large scale distributions of "long-lasting insecticide treated bed nets (LLIN), indoor residual spraying (IRS), intermittent presumptive treatment of pregnant women (IPT), and treatment with effective medicines, principally artemisinin-based 
combination therapy (ACT)" (Otten et al., 2009, 2). According to Otten et al.'s (2009) analysis, the Roll Back Malaria Initiative significantly lessened the prevalence of malaria in Ethiopia and Rwanda. However, despite all the progress that has been made to combat malaria, anthropogenic environmental alterations are changing the landscape of malaria prevalence in Ethiopia.

Like many other Sub-Saharan Africa countries, Ethiopia has been increasing its hydroelectric infrastructure and is planning new hydroelectric projects. The locations of Ethiopia's current hydroelectric dams as well as the locations of hydroelectric dams that are under construction is presented in Figure 1. According to the AQUASTAT Database and Global Reservoir and Dam Database (GRanD), there are currently eleven hydroelectric dams in Ethiopia; yet, twelve more hydroelectric dam projects are under construction or planned.

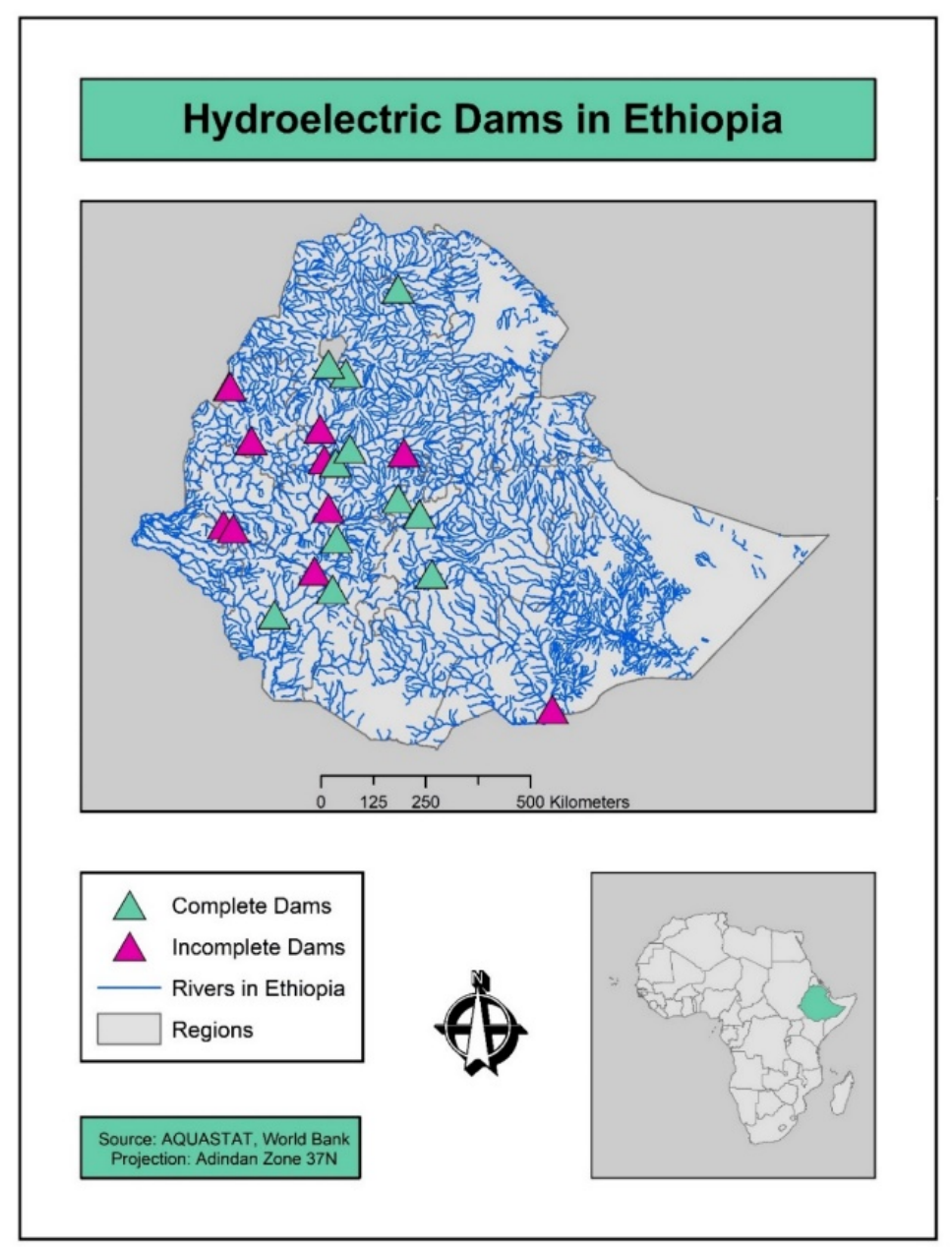

Figure 1. Current and Planned Hydroelectric Dams in Ethiopia.

Hydroelectric dams generate large amounts of energy. In countries like Ethiopia, that still has a large portion of their population lacking access to reliable energy, large hydroelectric dams are often viewed as a solution to energy paucity. Economic gains and energy production often take priority over the negative consequences of large hydroelectric. When large impoundment hydroelectric dams are built, a reservoir is created (Types of Hydropower Plants, n.d.). These reservoirs can hold substantial amounts of water and change the landscape of the terrain upstream of a dam. Dams slow down the flow of water which disrupts the natural flow. During drought periods, the water in reservoirs can become placid creating more breeding areas for mosquitos. Against this background, it makes it imperative to investigate how dams are changing the environment and ecosystem of Ethiopia, and to identify populations that are at an increased risk of malaria due to these changes. 
Scholarly literature establishes a positive relationship between proximity to dams and increased malaria risk in Sub-Saharan Africa (Ghebreyesus et al., 1999, Lautze et al., 2007, Yewhalaw et al., 2009). This makes malaria, which is already a significant health problem in the region, an even more prominent issue. Kibret et al., 2015, found that fifteen million people live within five kilometers of large dam reservoirs in Sub-Saharan Africa with 1.1 million malaria cases attributed to large dams each year. As Ethiopia continues to expand its hydroelectric infrastructure, it is essential that research focuses on identifying at-risk populations living in proximity of large dam reservoirs.

Large dams impact malaria rates because they change environments. Malaria is dependent on Anopheles mosquitos. Previous research has quantified at-risk populations based on proximity to reservoirs which results in a large total number of people being considered at increased risk of malaria. Remote sensing offers researchers the ability to identify mosquito breeding habitats. The purpose of this study is to locate mosquito breeding habitats near the [Gilgel Gibe III Hydroelectric Dam (Gibe III)] in Ethiopia and identify populations living within three kilometers of these habitats. Furthermore, the identification of mosquito breeding habitats will allow researchers to visualize the areas where anopheline larval control with larvicides and environmental measures would be most effective (Mushinzimana et al., 2006).

This research's four objectives were to utilize remote sensing techniques to locate mosquito breeding habitats, quantify how the Gibe III impacted mosquito breeding habitats, identify populations at greater risk of malaria due to the Gibe III and to compare techniques for identifying populations at greater risk of malaria due to hydroelectric dam.

\section{Literature Review}

Scholarly research has investigated the relationship between the built environment and increased malaria risk. The following studies specifically evaluate malaria prevalence in populations located at survey sites at differing distances from dams. A commonly used technique throughout the cited literature is regression analysis. It enables the researchers to evaluate how several possible independent variables help explain a dependent variable. The following studies utilize several types of regression analyses at large scales to evaluate the relationship between malaria and dams.

A study conducted by Yewhalaw et al. (2009) utilizes regression analysis, to examine the relationship between hydroelectric infrastructure and malaria in Ethiopia. Their methodology involves using logistic regression analyses based on survey results. A survey was taken "among children under 10 years of age living in three 'at-risk' villages (within $3 \mathrm{~km}$ ) and three 'control' villages (5 to $8 \mathrm{~km}$ )" (Yewhalaw et al., 2009, 3) around the Gilgel Gibe I Hydroelectric Dam in Southwestern, Ethiopia. Their results yield that "significantly higher malaria prevalence was observed in children living within three kilometers from the reservoir than those living farther away $(\mathrm{OR}=1.81,95 \% \mathrm{CI}=$ 1.17, 2.79 for P. vivax and OR = 2.57, 95\% CI = 1.01, 6.57 for P. falciparum)" (Yewhalaw et al., 2009, 4). These results show that villages located in closer proximity to the Gilgel Gibe I Hydroelectric Dam and reservoir tend to have higher risk of malaria prevalence.

Lautze et al. (2007) also examines the relationship between malaria and dams and reservoirs. Their study examines Kebeles located around the Koku reservoir in Ethiopia. They examine Kebeles (small administrative units in Ethiopia) at distances within three kilometers, between three kilometers and six kilometers, and between six kilometers and nine kilometers surrounding the Koku reservoir (Lautze et al., 2007, 982). They found that malaria prevalence was higher in the cohort located closest to the reservoir (within three kilometers). They also conduct spatial regression analysis which yielded significant results for all the variables examined which included proximity to reservoir, distance to malaria center, precipitation values for July to September, and precipitation values for October to December (Lautze et al., 2007, 986-987).

Understanding how mosquitos breed and how they respond to environmental changes is another component to understanding the relationship between hydroelectric dams and malaria prevalence. A study conducted by Ghebreyesus et al. (1999), examines the relationship between malaria and microdams in the Tigray region of Northern Ethiopia. These dams are much smaller than large hydroelectric dams, but they still change the natural landscape and flow of water. The authors 
also implement multivariate regression analysis. They identified eight microdams and surveyed "about 7000 children under 10 years living in villages within $3 \mathrm{~km}$ of [the] microdams and in control villages 8-10 km distant" (Ghebreyesus et al., 1999, 663). Their results found "a 7.3-fold increased [malaria] incidence close to dams. This is a highly significant difference, with a 95\% confidence interval of 4.7 to 11.3" (Ghebreyesus et al., 1999, 664). This study affirms even small dams and reservoirs can significantly impact malaria prevalence within vulnerable members of nearby populations.

Remote sensing technologies offer health researchers an alternative way to study the geography of diseases. Remote sensing technologies have been used to monitor disease and create risk maps since the late 1990s (Beck et al., 2000). Remote sensing enables researchers to acquire information about a location without physical contact or conducting laborious amounts of field work. It also enables research to be conducted in areas with limited available spatial data. Several different types of devices are used to collect remotely sensed data. One of the most prevalent devices are satellites. Satellites are semi-permanent pieces of equipment that are continuously capturing imagery of Earth's surface. Satellite images cover large swaths of Earth's surface. The following studies discuss the implementation of satellite imagery to study malaria.

Beck et al. (2000), discuss how researchers use remote sensing data to study disease. This study discusses applications of disease mapping using satellite systems that were active when this paper was published. It cites six different studies that utilize remote sensing to map malaria vectors. The use of remote sensing to study vector disease and malaria has evolved over the past two decades. As Beck et al. (2000) predicted, the spatial, temporal, spectral, and radiometric resolution of satellites platforms have greatly improved. Remote sensing data is now more detailed and accessible.

A more recently published paper written by Ebhuoma and Gebreslasie (2016) evaluates how researchers use remote sensing to derive climatic and environmental variables essential for modeling malaria transmission in Sub-Saharan Africa. They divided Sub-Saharan Africa into four regions (East, West, Central, and Southern). They searched for commonalities between researchers' variables and source of remote sensing data sources. Ethiopia is located within the East Africa region. The statistically significant remote sensing variables found within the East Africa region were: [Normalized Difference Vegetation Index (NDVI)], [Land Surface Temperature (LST)], [Rainfall Estimates (RFE)], [Environmental Vegetation Index (EVI)], Elevation/Altitude, and [Land Use and Land Cover (LULC)].

In 2006, Mushinzimanan et al. (2006) published a study that evaluates Anopheles mosquito larval habitats and discusses how remote sensing can be used to identify them in western Kenya. A [Digital Elevation Model (DEM)] was used to "extract topographic parameters that may be associated with mosquito larval habitat formation, such as elevation, wetness index, flow distance to stream, aspect of land-surface and curvature" (Mushinzimanan et al., 2006, 3). This data was combined with a supervised classification to determine areas suitable for mosquito breeding.

An even more recent study conducted by Minale and Alemu (2018), focuses on creating a malaria risk map for Bahir Dar City, Amhara Province in northeast Ethiopia. Their risk map was derived from "temperature, rainfall, terrain (altitude and slope), proximity-related factors (distance from lake, river, and health facilities) and LULC" (Minale and Alemu, 2018, 159). Each variable was weighted based on local knowledge and prior research.

An approach utilized throughout scholarly literature for identifying at-risk populations for malaria due to the presence of large dams is the calculation of buffer distances around a reservoir, and analysis of the total number of people living within that area. A comprehensive study conducted by Kibret et al. (2015) quantifies the total number of people in Sub-Saharan Africa at-risk of malaria due to the presence of large dams. Their research uses a five-kilometer buffer around each reservoir. This buffer distance was determined by the author's estimated flight range of mosquitos.

Another study uses a similar technique for locating at-risk populations. Keiser et al. (2005) examined hydroelectric dam reservoirs in Sub-Saharan Africa. Their technique involves approximating the size of each reservoir as a rectangle; then, locating all areas within two kilometers 
of the reservoir. Keiser et al. (2005) justifies using a two-kilometer buffer based on the estimated flight range of a mosquito.

\section{Materials and Methods}

\subsection{Study Area}

The study area is in southwestern Ethiopia. Ethiopia's 2018 estimated population was 107.53 million, which places it as the fourteenth most populous country in the world (Ethiopia Population 2018 , n.d.). Ethiopia's population has high rates of poverty and a low income per capita (Ethiopia Population 2018, n.d.). The combination of high poverty, economic insecurity, and endemic malaria makes Ethiopia's population susceptible to malaria mortality.

This research examined mosquito breeding habitats and populations at-risk of malaria near the Gibe III's reservoir. The Gibe III is located on the Omo River in the Southern Nations, Nationalities, and Peoples' Region in southwestern Ethiopia. Construction began on the Gibe III in 2006. It is the third hydroelectric infrastructure project and second dam located on the Gilgel Gibe and Omo Rivers. The Gilgel Gibe I Dam is located on the Gilgel Gibe River approximately 108 kilometers North of the Gibe III (measurements were taken in Google Earth). It was built to divert water to the Gilgel Gibe II Powerstation that is located 27 miles Southeast of the Gibe I dam. Two more large hydroelectric dams are planned to be built on the Gilgel Gibe River. These hydroelectric projects are significantly changing the landscape of the Omo and Gilgel Gibe river valleys. The Gibe III's reservoir, at capacity, can hold 14,700 million cubic meters of water (Poindexter, 2015). When the Gibe III was in construction, multiple organizations including the United Nations attempted to stop it from being finished. They cited concerns for the ecosystem of Lake Turkana in Kenya, the largest desert lake in Africa. Despite these concerns, the Gibe III was completed and began generating electricity in 2015.

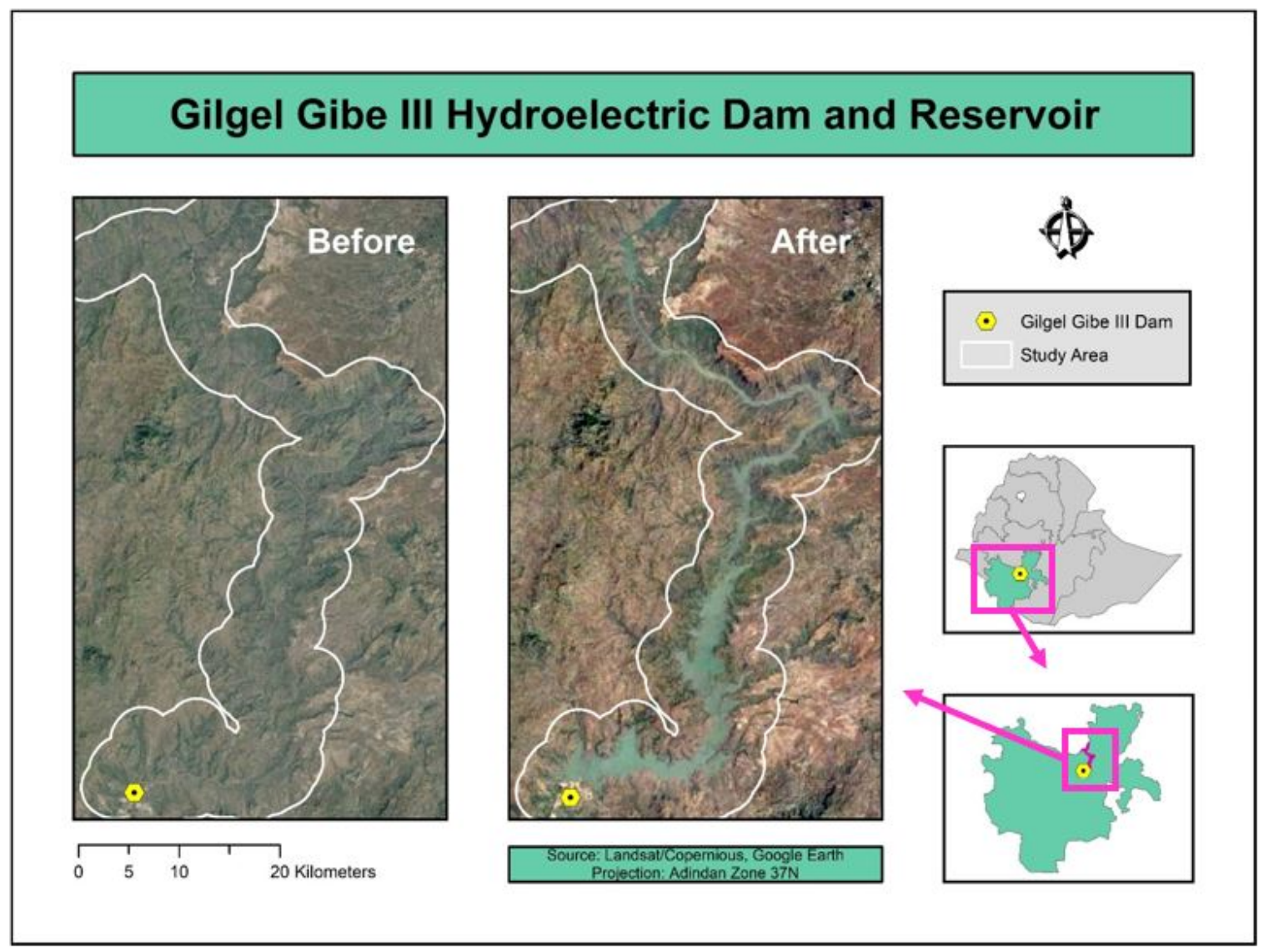

Figure 2. Study Area Before and After the Gibe III Hydroelectric Dam 
The study area, as denoted in the maps featured in Figure 2, includes all areas within three kilometers of the Gibe III's reservoir. It is $1055.416 \mathrm{~km} 2$. Three kilometers was chosen based on the flight range of mosquitos. Scholarly research documents that mosquitos tend to have maximum flight ranges. Their findings vary from two kilometers to five kilometers. Three kilometers was chosen for this study because of the findings of the Yewhalew et al. (2006) study, that was conducted around the Gilgel Gibe I reservoir. Their findings showed statistically significant malaria prevalence in villages located within three kilometers of the reservoir compared to villages located further away.

\subsection{Data}

The data sources are listed in Table 1. The Landsat 8 satellite imagery, [Shuttle Radar Terrain Mission (SRTM)] DEM, and Sentinel 2 satellite imagery were downloaded from the United States Geological Survey (USGS) (https://earthexplorer.usgs.gov/). The Landscan High Resolution Global Population Dataset was downloaded from Oakridge National Laboratory (https://landscan.ornl.gov/). All the input data used in the risk models can be freely downloaded or manually created. This allows the model to be adapted for other study areas.

Table 1. Data Sources

\begin{tabular}{cccc}
\hline Data & Source & Details & Spatial Resolution \\
\hline Landsat 8 Satellite & USGS - Earth & Imagery from before the Gibe \\
Imagery & Explorer & III was completed (December \\
& 11, 2014) & $30 \mathrm{~m}$ \\
Landsat 8 Satellite & USGS - Earth \\
Imagery & Explorer & $\begin{array}{c}\text { Imagery from after the Gibe } \\
\text { III was completed (December } \\
\text { 3, 2017) }\end{array}$ & $30 \mathrm{~m}$ \\
SRTM - DEM & USGS - Earth & $\begin{array}{c}\text { GeoTiff DEM } \\
(2000)\end{array}$ & $30 \mathrm{~m}$ \\
Sentinel 2 Satellite & USGS - Earth & Imagery from after the Gibe & $20 \mathrm{~m}$ \\
Imagery & Explorer & III was completed \\
& (December 23, 2017) & \\
Landscan High & Oakridge & Raster dataset with ambient & $1 \mathrm{~km}$ \\
Resolution Global & National & population estimates \\
Population Dataset & Laboratory & (2012, 2013, 2014, 2015, 2016, & \\
& & 2017) & \\
\hline
\end{tabular}

\subsection{Methods}

The first research objective was to identify probable mosquito breeding habitats using remotely sensed data. ArcGIS 10.6.1 Model-Builder was utilized to automate the creation of a mosquito breeding habitat risk map. Two versions of the model were created to examine before and after the Gibe III was completed. The model extracts all input data to the study area, calculates and creates variables, reclassifies each variable, and overlays the variables with assigned ratings, scales, and weights. Table 2 list the four variables used in the model: Slope, Elevation, LST, and [Modified Normalized Difference Water Index (MNDWI)] with assigned scale and weight. These variables were selected because scholarly literature established statistically significant relationships between them and mosquito breeding.

The first step of the model was to extract the input data (Landsat 8 bands and STRM DEM) used to create the four variables to the study area. To accomplish this, the Gibe III reservoir was manually digitized using Sentinel 2 imagery from December 23, 2017. Then, a three-kilometer buffer was created around the reservoir to create the study area. All input data was extracted by the study area. 
Table 2. Criteria, Rating, Scales and Weights for Mosquito Breeding Habitat Risk Model

\begin{tabular}{ccccccc}
\hline Criteria & $\begin{array}{c}\text { Low- } \\
\text { Risk } \\
\mathbf{( 1 )}\end{array}$ & $\begin{array}{c}\text { Low/Medium- } \\
\text { Risk } \\
\mathbf{( 2 )}\end{array}$ & $\begin{array}{c}\text { Medium- } \\
\text { Risk } \\
\mathbf{( 3 )}\end{array}$ & $\begin{array}{c}\text { Medium/High- } \\
\text { Risk } \\
\mathbf{( 4 )}\end{array}$ & $\begin{array}{c}\text { High- } \\
\text { Risk } \\
\mathbf{( 5 )}\end{array}$ & $\begin{array}{c}\text { Assigned } \\
\text { Weight }\end{array}$ \\
\hline Slope & $>20.1^{\circ}$ & & $15.1^{\circ}-20^{\circ}$ & $7.1^{\circ}-15^{\circ}$ & $<7^{\circ}$ & $7 \%$ \\
Elevation & $>2500$ & $1500-$ & & $<1500$ & $11 \%$ \\
(Meters) & & 2500 & & & \\
MNDWI & $<0.1$ & $0.2-1$ & & $24^{\circ}-28^{\circ}$ & $41 \%$ \\
LST & $<16^{\circ}$, & $16^{\circ}-24^{\circ}$, & & \\
(Celsius) & $>40^{\circ}$ & $28^{\circ}-40^{\circ}$ & & & $41 \%$ \\
\hline
\end{tabular}

The slope of terrain determines where water accumulates. Slope was calculated using the SRTM DEM and the Slope tool in ArcGIS 10.6. The SRTM DEM was captured in 2000. Therefore, the slope for both time periods was the same. The study area has slopes ranging from $0^{\circ}$ to $75.418^{\circ}$. Slope was reclassified within the model into four risk categories. The break values for the slope reclassification were chosen based on the research of Alemu and Minale (2018): High-Risk (0-7), Medium/High-Risk (7-15), Medium-Risk (15-20), and Low-Risk (> 20).

Elevation is another critical criterion and constraint to consider when evaluating mosquito breeding habitats. Temperature is a key component for mosquito breeding. As elevations increase, temperatures decrease. Scholarly research has documented that mosquito breeding is significantly affected by elevation. In Sub-Saharan Africa, research documents limited mosquito breeding occurs in elevations greater than 1500 meters and low to no mosquito breeding occurs at elevation greater than 2500 meters (Minale and Alemu, 2018). The study area elevations were acquired directly from the SRTM DEM. Elevation values for the study area range from 672 to 2031 meters above sea level. Elevation values were reclassified into three risk categories, shown in Table 2, High-Risk $(<1500$ meters), Medium-Risk (1500 - 2500 meters), and Low-Risk (> 2500 meters)

LST was calculated using Landsat 8 imagery from before and after the Gibe III was completed. Calculating LST requires the calculation of several intermediate variables (Sim, 2017). It uses Landsat 8 bands 4, 5, and 10. All raster calculations were done using the Raster Calculator in ArcGIS 10.6.1. First, [Top of Atmosphere (TOA)] reflectance was calculated using the formula:

$$
\mathrm{TOA}(\mathrm{L})=\mathrm{ML} * \mathrm{Qcal}+\mathrm{AL}
$$

where: $\mathrm{M}_{\mathrm{L}}=$ Band-specific multiplicative rescaling factor from the metadata

(RADIANCE_MULT_BAND_x, where $\mathrm{x}$ is the band number)

Then, TOA was converted to [Brightness Temperature (BT)] using the formula:

$$
\mathrm{BT}=(\mathrm{K} 2 /(\ln (\mathrm{K} 1 / \mathrm{L})+1))-273.15
$$

where: $\mathrm{K} 1=$ Band-specific thermal conversion constant from the metadata

(K1_CONSTANT_BAND_x, where $\mathrm{x}$ is the thermal band number).

$\mathrm{K} 2=$ Band-specific thermal conversion constant from the metadata

(K2_CONSTANT_BAND_x, where $\mathrm{x}$ is the thermal band number)

BT is one of the input variables used to calculate the LST. Another input variable that is required to calculate LST is [Emissivity $(\varepsilon)$ ]. First, a NDVI was calculated and converted to the [Proportion 
Vegetation (PV)]. The NDVI formula converts all pixels in the study area into values ranging from 1 to +1 . The more negative the NDVI value (closer to -1 ), the less healthy vegetative landcover. The more positive the NDVI value (closer to +1 ), the healthier vegetative landcover. NDVI was calculated using the formula:

$$
\mathrm{NDVI}=(\mathrm{NIR}-\mathrm{Red}) /(\mathrm{NIR}+\mathrm{Red})
$$

where: In Landsat 8 imagery, Near Infrared (NIR) reflectance is band 5 and Red reflectance is band 4 .

The NDVI output raster was then converted in PV using the formula:

$$
\mathrm{Pv}=\text { Square ((NDVI - NDVImin) / (NDVImax - NDVImin)) }
$$

where NDVImin is equal to the minimum value in the NDVI raster and NDVImax is equal to the maximum value in the NDVI raster.

For the before image, the minimum NDVI value was -0.252322 and the maximum NDVI value was 0.559816 . For the after image, the minimum NDVI value was -0.175683 and the maximum NDVI value was 0.556848 .

Next, $\varepsilon$ was calculated using the formula:

$$
\varepsilon=0.004 * \mathrm{Pv}+0.986
$$

Where 0.986 corresponds to a correction value for the equation.

Then, LST was calculated using the formula:

$$
\mathrm{LST}=(\mathrm{BT} /(1+0.00115 * \mathrm{BT} / 1.4388) * \operatorname{Ln}(\varepsilon)))
$$

To evaluate how the Gibe III changed mosquito breeding habitats, LST values were reclassified into consistent classes for both time periods. The classes were determined, and applied scale values based on Anopheles mosquito breeding considerations. Beck-Johnson et al. (2013) models and identifies the ideal temperatures at which Anopheles mosquitos breed. They found between $24^{\circ}$ Celsius and $28^{\circ}$ Celsius the largest amount of Anopheles larva survives. The MNDWI was created by Hanqiu $\mathrm{Xu}$ in 2006. Its purpose is to discriminate water features from built-up land (Xu, 2006). It is a modification of the [Normalized Difference Water Index (NDWI)]. The NDWI utilizes [Near Infrared (NIR)] and Green bands. It works sufficiently well but has limitations discriminating between built-up land features and open water due to these landcovers having similar NIR reflectance values. Xu identified this problem and proposed using a [Middle Infrared (MIR)] band instead. The MNDWI is calculated using the formula:

$$
\text { MNDWI }=\text { Green }- \text { MIR } / \text { Green }+ \text { MIR }
$$

In Landsat 8 imagery, Green reflectance is band 3 and SWIR is band 6. Band 6 is used as the MIR band.

The MNDWI calculates values per pixel ranging from -1 to +1 . The closer the MNDWI value is to +1 the more undisturbed water is present.

MNDWI is used as a proxy for LULC. Wetlands and water landcovers positively contribute to mosquito breeding (Minale and Alemu, 2018). Through manual examination, MNDWI values for wetland areas were sampled throughout the study area. Wetland areas tended to have a MNDWI values ranging from 0.1 to 0.2 . The MNDWI raster was reclassified into three classes: -1 to 0.1 (Nonwater or wetland landcovers), $0.1-0.2$ (Wetlands and/or slow-moving water), 0.2 to 1 (Open water).

The four variables were combined using the Weighted Overlay tool in ArcGIS 10.6.1. Weights were assigned using two methods: statistical significance established in scholarly literature and the Analytical Hierarchical Process. Analytical Hierarchical Process is a technique used to determine 
multi-criteria weighting schemes (Vargas, 2012). An Analytical Hierarchical Process calculator developed by Klaus D. Goepel (2013) was utilized. It allows users to input their criteria to conduct a pairwise comparison. The Weight Overlay tool requires the user to select an evaluation scale, assign scale values to the classes of each input raster, and a weight to each input raster. A 1 to 5 by 1 evaluation was selected for this research. The scale and weights for each variable are listed in Table 2.

To evaluate how the Gibe III has changed mosquito breeding habitats in the study area, the mosquito breeding habitat risk model was applied with the variables of before and after the Dam construction. Outputs contain attribute information that includes the number of pixels classified in each of the five risk categories. The total area of each risk category, the percentage of the study area, and the difference between the two time periods were calculated and compared.

To identify populations living near High-Risk mosquito breeding habitats, the study located concentrations of High-Risk mosquito breeding pixels. Outputs of the mosquito breeding habitat risk models contained isolated pixels throughout the study area. The focus of this research was to identify populations that were at-risk of malaria. Therefore, it was necessary to clean the model outputs to eliminate misclassified and isolated pixels. This was accomplished with the Boundary Clean tool in ArcGIS 10.6.1. All data used in this research were raster datasets. To locate populations living within three kilometers of the main concentrations of High-Risk areas for mosquito breeding, the model outputs had to be converted into vector data. This allowed for areas with concentrations of High-Risk pixels to be manually selected, exported, and buffered using ArcGIS 10.6.1. The population was quantified within three kilometers of the two concentrated High-Risk areas.

The results from the remote sensing-based approach were compared with a proximity approach. Scholarly literature has measured all populations living within a set distance from a reservoir and identified them as being at-risk for malaria (Keiser et al., 2000, Kibret et al., 2015). Proximity based approach assumes that mosquito breeding habitats are equally distributed within the reservoir. A proximity analysis population risk map was generated for the entire study area and compared to the remote sensing approach risk maps.

\section{Results}

The four variables (slope, elevation, LST, and [Modified Normalized Difference Water Index (MNDWI)] used in the model were extracted. The outputs are presented in Figure A1, Figure A2, Figure A3 and Figure A4 in the section of Appendix.

As a result, mosquito habitat risk maps were created. The models were used to evaluate two time periods: before and after the Gibe III was completed. The two outputs of the mosquito habitat risk models are featured in Figure 3. Before the Gibe III was finished, more of the study area is classified as Low/Medium and Low Risk. After the Gibe III dam was completed, more portions of the study area are High, Medium/High and Medium Risk. In the After map in Figure 3, all the High-Risk areas occur on the edges of the Gibe III's reservoir. This reservoir did not exist until the Gibe III was completed.

There are two main concentrations of High-Risk areas within the study area. The largest of these occurs directly northeast of the Gibe III. It is denoted by the red box labeled 1 in Figure 3. In this area, the edges of the reservoir are identified as High-Risk; while, the middle of the reservoir is identified as Medium/High-Risk. The second primary concentration area occurs midway in the reservoir. It is highlighted by the red box labeled 2 in Figure 3.

According to the mosquito habitat risk models, the Gibe III increased severity of mosquito breeding habitat risk in the study area. Before the Gibe III was completed, only $0.31 \%$ of the study area was classified as High-Risk and $0.39 \%$ as Medium/High-Risk. After the dam was completed, $2.37 \%$ of the image was classified as High-Risk and $5.36 \%$ as Medium/High-Risk. The total area, difference between the two time periods, and percentages of the study area for each risk category are listed in Table 3. 


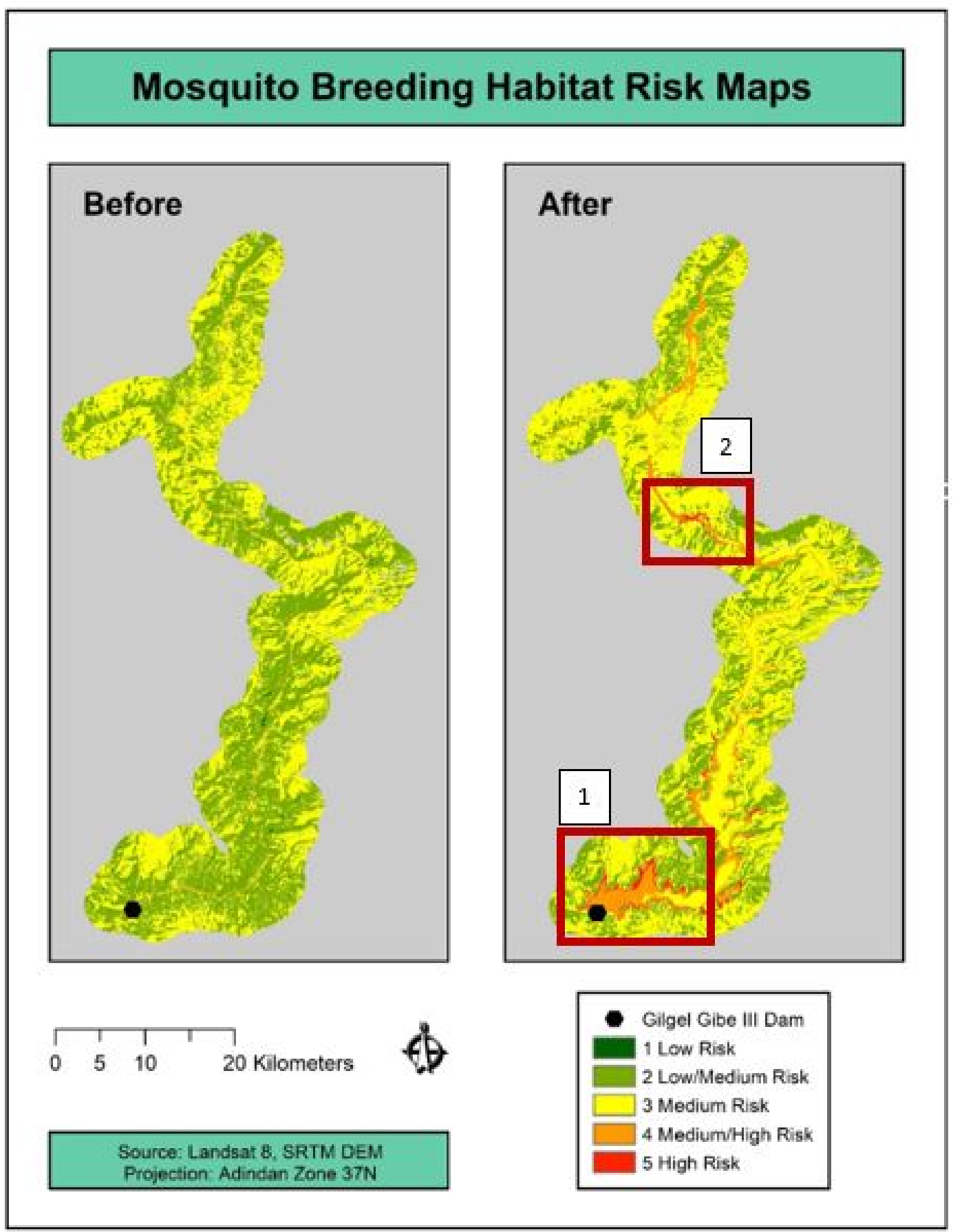

Figure 3. The Mosquito Breeding Habitat Risk Models

This research concentrated on developing a remote sensing approach for locating populations at-risk of malaria. As previously stated, there are two main High-Risk areas in the study area. According to the model, these two areas would pose the greatest risk for mosquito breeding habitats. The 2017 Landscan estimated ambient population living within three kilometers of these two areas is 22,569 . The Remote Sensing Approach map is featured in Figure 4. The two risk areas are outlined in red.

The Proximity Approach map shown in Figure 4, identifies all populations living within three kilometers of the Gibe III reservoir as being at-risk. The study area encompasses the same area as the proximity approach. The 2017 Landscan estimated ambient population risk area identified by the proximity approach is 51,987. The proximity approach identified 29,418 more people as being at-risk than the remote sensing approach. 
Table 1: Area, Difference, and Percentages of the Study Area for Each Risk Category

\begin{tabular}{|c|c|c|c|c|c|}
\hline Pixel Classification & $\begin{array}{c}\text { Before } \\
\text { (Area km2) }\end{array}$ & $\begin{array}{c}\text { After } \\
\text { (Area km2) }\end{array}$ & $\begin{array}{l}\text { Difference } \\
\text { (Area km2) }\end{array}$ & $\begin{array}{c}\text { Before } \\
\text { (\% of Study } \\
\text { Area) }\end{array}$ & $\begin{array}{c}\text { After } \\
\text { (\% of Study } \\
\text { Area) }\end{array}$ \\
\hline $\begin{array}{l}\text { 5: High } \\
\text { Risk }\end{array}$ & 3.27 & 25.01 & $\begin{array}{c}21.74 \\
\text { (more After) }\end{array}$ & $0.31 \%$ & $2.37 \%$ \\
\hline $\begin{array}{l}\text { 4: Medium/High } \\
\text { Risk }\end{array}$ & 4.12 & 56.57 & $\begin{array}{c}52.45 \\
\text { (more After) }\end{array}$ & $0.39 \%$ & $5.36 \%$ \\
\hline $\begin{array}{l}\text { 3: Medium } \\
\text { Risk }\end{array}$ & 484.75 & 619.32 & $\begin{array}{c}134.57 \\
\text { (more After) }\end{array}$ & $45.93 \%$ & $58.68 \%$ \\
\hline $\begin{array}{c}\text { 2: Low/Medium } \\
\text { Risk }\end{array}$ & 562.22 & 354.52 & $\begin{array}{c}207.7 \\
\text { (more Before) }\end{array}$ & $53.27 \%$ & $33.59 \%$ \\
\hline 1:Low Risk & 0.95 & $<0.01$ & $\begin{array}{c}0.95 \\
\text { (more Before) }\end{array}$ & $0.09 \%$ & $<0.01 \%$ \\
\hline
\end{tabular}

\section{Populations at Risk of Malaria}
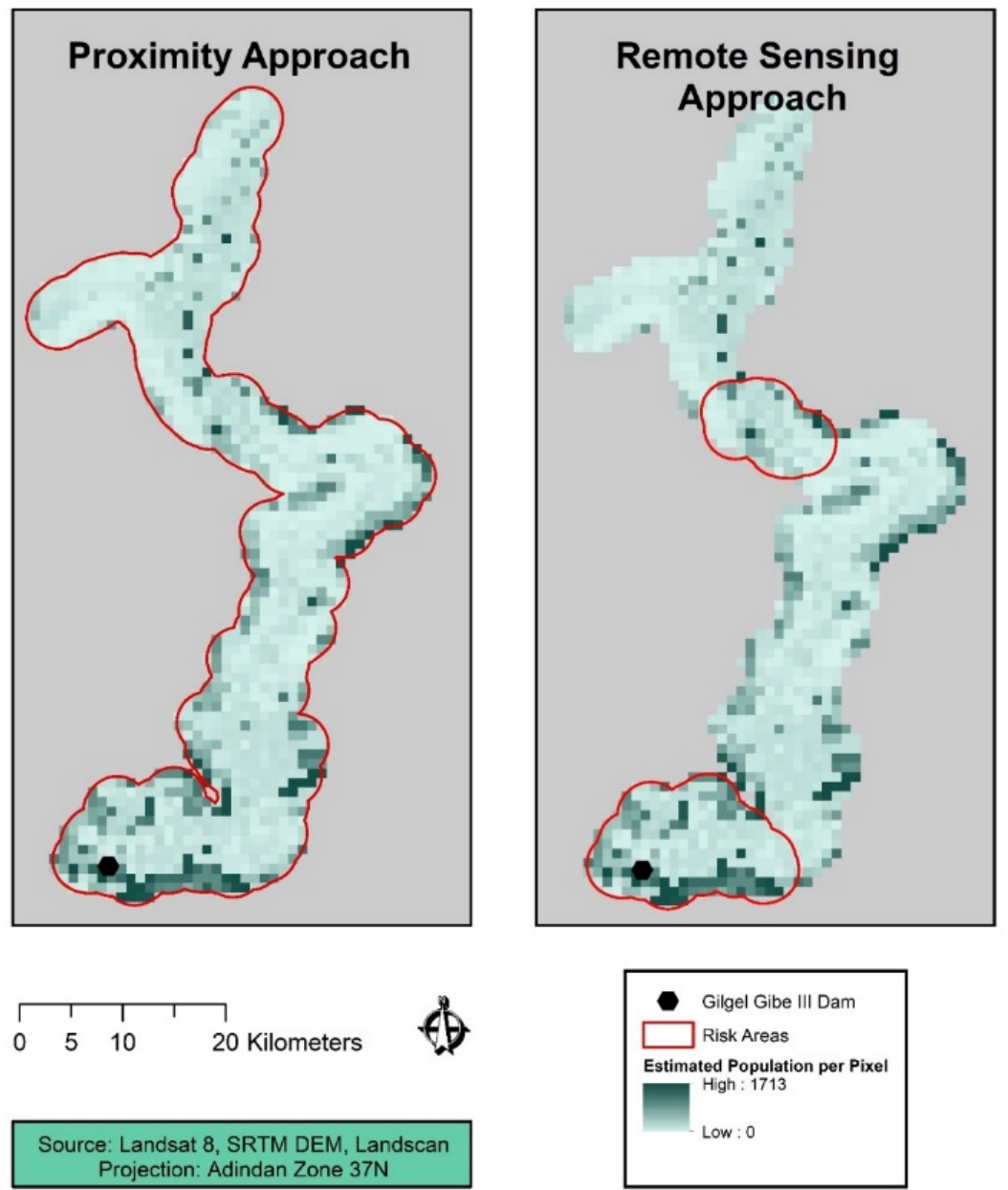

Gilgel Gibe III Dam Risk Areas Estimated Population per Pixel High : 1713 Low : 0

Figure 4. Estimated Population at Greater risk of Malaria 


\section{Discussion and Conclusion}

Large hydroelectric dams offer developing countries economic incentives and a path to energy independence; yet, there are major consequences to permanently altering the environment. The construction and completion of the Gibe III in Ethiopia caused a major displacement of people as shown by the graph in Figure A5 in the section of Appendix. Thousands of people were forced to relocate when the Gibe III reservoir was flooded.

The Gibe III also altered the landscape of the study area. According to the models created in this research, the Gibe III increased mosquito breeding habitat risk. It introduced the potential for 14,300 $\mathrm{m} 3$ of water to be housed within the study area. This created more water in low slope areas at elevations and LSTs conducive for mosquito breeding. The malaria breeding habitat risk models showed an increase in High-Risk, Medium/High-Risk, and Medium-Risk mosquito breeding habitats after the Gibe III was completed. Before the Gibe III, the High-Risk areas occurred along the edges of the Omo River. After the Gibe III, the High-Risk areas occur along the edges the Gibe III's reservoir primarily in sloughs and meanders.

Two approaches were conducted to identify populations at increased risk of malaria due to the Gibe III. The proximity approach was recreated based on techniques used by other researchers. It estimates that all people living within three kilometers of the Gibe III are at increased risk of malaria. For 2017, the proximity approach identified an estimated 51,987 people of being at-risk. This approach assumes that proximity to a reservoir is the only factor contributing to malaria risk.

The remote sensing approach seeks to locate where mosquitos are likely to breed first; then, it identifies the populations living near those area. While, there were isolated High-Risk areas along the edges of the Gibe III reservoir, two primary areas were identified. The largest is located directly upstream of the Gibe III. This area also has the densest estimated population in the study area living within three kilometers. The second area occurs midway in the reservoir. The areas within three kilometers of it is less densely populated than the first area. In total, the remote sensing approach identified 22,569 people at being at-risk of malaria which is 29,418 less than the proximity approach.

The two approaches discussed in this research offer different perspectives and represent different degrees of risk. The proximity approach has been statistically proven at other study sites. It is representative of general risk. The remote sensing approach is a more targeted approach that was developed based on previous research to identify the populations at the highest risk. The proximity approach and the remote sensing approach should be used in conjuncture with one another.

For the people living in the study area, the Gibe III could affect their health and livelihood long term. Remote Sensing and Geographic Information Systems (GIS) technologies allow researchers to identify where the changes in environments and locate populations at-risk because of environmental changes. In rural communities where aid and resources are often limited, it is essential that the people that are at the greatest risk can identified and prioritized.

Furthermore, the results of this research could be used to hold the parties responsible for the increasing risk accountable. As previously discussed, as Ethiopia expands their hydroelectric infrastructure the two approaches presented in this research could also be utilized to predict risk near planned dams.

One of the limiting factors of this research was the resolution of the data. Free, accessible satellite imagery tends to have coarse spatial resolution. The Landsat 8 and SRTM DEM data has a spatial resolution of 30 meters. The Landscan population estimate data is one kilometer. This means that some specific information and details may not have been represented. Future research could invest into purchasing higher resolution imagery. Despite the limitations that low-resolution data can cause, it is essential for scientific research. These data sources were used so that the model could be easily modified and replicated for other study areas. All the models input data can be freely downloaded or easily replicated.

Another limitation is the lack of field work and malaria survey data. This limits the ability to test the statistical significance of the mosquito breeding habitat risk model. Field work is an essential element in medical geography studies. Future research should focus on developing a malaria 
prevalence survey and ground truth testing of the High-Risk mosquito breeding habitats identified by the model.

Despite these limitations, studies of this nature are important because of the lack of spatial data available in Ethiopia. As major landscape alterations are occurring throughout Sub-Saharan Africa, it is paramount that the relationship between malaria and dams continues to be researched.

Author Contributions: Conceptualization, A.S. and S.S.; data collection, A.S.; Formal analysis, A.S.; investigation, A.S.; methodology, A.S. and S.S.; resources, A.S. and S.S.; writing - original draft, A.S., writing review, editing and revision, S.S.; All authors have read and approved the final manuscript.

Funding: This research is partially funded by the University of North Alabama College of Arts and Sciences.

Conflicts of Interest: The authors declare no conflict of interest.

\section{References}

AQUASTAT Database http://www.fao.org/aquastat/en/databases/dams/

Ayele DG, Zewotir TT, Mwambi HG, 2012. Prevalence and Risk Factors of Malaria in Ethiopia. Malar J 11:195

Beck LR, Lobitz BM, Wood BL, 2000. Remote Sensing and Human Health: New Sensors and New Opportunities. Emerg Infect Dis 6:217-227.

Beck-Johnson LM, Nelson WA, Paaijmans KP, Read AF, Thomas MB, Bjørnstad ON, 2013. The Effect of Temperature on Anopheles Mosquito Population Dynamics and the Potential for Malaria Transmission. PLoS ONE 8:e79276.

Centers of Disease Control and Prevention, 2018. Malaria. Available from: https://www.cdc.gov/parasites/malaria/index.html Accessed: October 2018.

Ebhuoma O, Gebreslasie M, 2016. Remote Sensing-Driven Climatic/Environmental Variables for Modelling Malaria Transmission in Sub-Saharan Africa. Int. J. Environ. Res. Public Health 13:584.

Ethiopia Population 2018. n.d. Total Population by Country 2018. Available from: worldpopulationreview.com/countries/ethiopia-population/ Accessed: March 2019.

Ghebreyesus TA, Mitiku H, Witten KH, Getachew A, Yohannes AM, Yohannes M, Teklehaimanot HD, Lindsay SW, Byass P, 1999. Incidence of Malaria among Children Living near Dams in Northern Ethiopia: Community Based Incidence Survey. BMJ 319:663-666.

Gilgel Gibe III Dam, Ethiopia. n.d. Environmental Justice Atlas. Available from: https://ejatlas.org/conflict/gibeiii-dam-ethiopia Accessed: October 2018.

Global Reservoir and Dam Database (GRanD) http://globaldamwatch.org/grand/

Goepel, K.D. Implementing the Analytic Hierarchy Process as a Standard Method for Multi-Criteria Decision Making In Corporate Enterprises-A New AHP Excel Template with Multiple Inputs. In Proceedings of the International Symposium on the Analytic Hierarchy Process 2013, Kuala Lumpur, Malaysia, 23-26 June 2013.

Grand Ethiopian Renaissance Dam (GERD) in Eight Years. 2019. Ethiopian News Agency. Available from: https://www.ena.et/en/?p=7207 Accessed: May 2019.

Hay SI, Guerra CA, Tatem AJ, Noor AM, Snow RW, 2004. The Global Distribution and Population at Risk of Malaria: Past, Present, and Future. Lancet Infect Dis 4:327-336.

Keating J, MacIntyre K, Mbogo C, Githeko A, Regens JL, Swalm C, Ndenga B, Steinburg LJ, Kibe L, Githure JI, Beier JC, 2003. A Geographic Sampling Strategy for Studying Relationships between Human Activity and Malaria Vectors in Urban Africa. Am J Trop Med Hyg 68:357-365.

Keiser J, De Castro MC, Maltese MF, Bos R, Tanner M, Singer BH, Utzinger J, 2005. Effect of Irrigation and Large Dams on the Burden of Malaria on a Global and Regional Scale. Am J Trop Med Hyg 72:392-406. 
Kibret S, Lautze J, McCartney M, Wilson GG, Nhamo L, 2015. Malaria Impact of Large Dams in Sub-Saharan Africa: Maps, Estimates and Predictions. Malar J 14:339.

Landscan, https://landscan.ornl.gov/

Lautze J, McCartney M, Kirshen P, Olana D, Gayathree J, Spielman A, 2007. Effect of a Large Dam on Malaria Risk: The Koka Reservoir in Ethiopia. Trop Med Int Health 12:982-989.

Otten M, Aregawi M, Were W, Karema C, Medin A, Bekele W, Jima D, Gausi K, Komatsu R, Korenrom E, LowBeer D, Grabowsky M, 2009. Initial Evidence of Reduction of Malaria Cases and Deaths in Rwanda and Ethiopia Due to Rapid Scale-up of Malaria Prevention and Treatment. Malar J 8:14.

Minale AS, Alemu K, 2018. Mapping Malaria Risk Using Geographic Information Systems and Remote Sensing: The Case of Bahir Dar City, Ethiopia. Geospat Health 12:157-163.

Mushinzimana E, Munga S, Minakawa N, Li L, Feng C, Bian L, Kitron U, Schmidt C, Beck L, Zhou G, Githeko AK, Yan G, 2006. Landscape Determinants and Remote Sensing of Anopheline Mosquito Larval Habitats in the Western Kenya Highlands. Malar J 5:13.

Poindexter GB, 2015. Power Generation Begins at 1,870 MW Gibe III Hydroelectric Project in Ethiopia. Hydro Review. Available from: https://www.hydroworld.com/articles/2015/10/power-generation-begins-at-1870-mw-gibe-iii-hydroelectric-project-in-ethiopia Accessed: May 2019.

Sim S, 2017. Social vulnerability to heat in Greater Atlanta, USA: spatial pattern of hear, NDVI, socioeconomics and household composition. Proc. SPIE 10413, Remote Sensing Technologies and Application in Urban Environments II, 1043105. October 2017.

Types of Hydropower Plants, n.d. Office of Energy Efficiency and Renewable Energy. U.S. Department of Energy. Retrieved May 5, 2019. Available from: https://www.energy.gov/eere/water/typeshydropower-plants Accessed: March 2019.

The GERD Support Team in Vancouver and Victoria, BC, Canada. n.d. The GERD Support Team in Vancouver and Victoria, BC, Canada. Available from: ethiopiangranddam.wordpress.com/ Accessed: April 2019.

USGS Earth Explorer. https://earthexplorer.usgs.gov/

Vargas RV, 2010. Using the analytic hierarchy process (ahp) to select and prioritize projects in a portfolio. Paper presented at PMI® Global Congress 2010-North America, Washington, DC. Newtown Square, PA: Project Management Institute.

$\mathrm{Xu} \mathrm{H}, 2006$. Modification of Normalised Difference Water Index (NDWI) to Enhance Open Water Features in Remotely Sensed Imagery. Int J Remote Sens 27: 3025-3033.

Yewhalaw D, Worku L, Wim VB, Gebre-Selassie S, Kloos H, Duchateau L, Niko S, 2009. Malaria and Water Resource Development: The Case of Gilgel-Gibe Hydroelectric Dam in Ethiopia. Malar J 8:21. 


\section{Appendix}
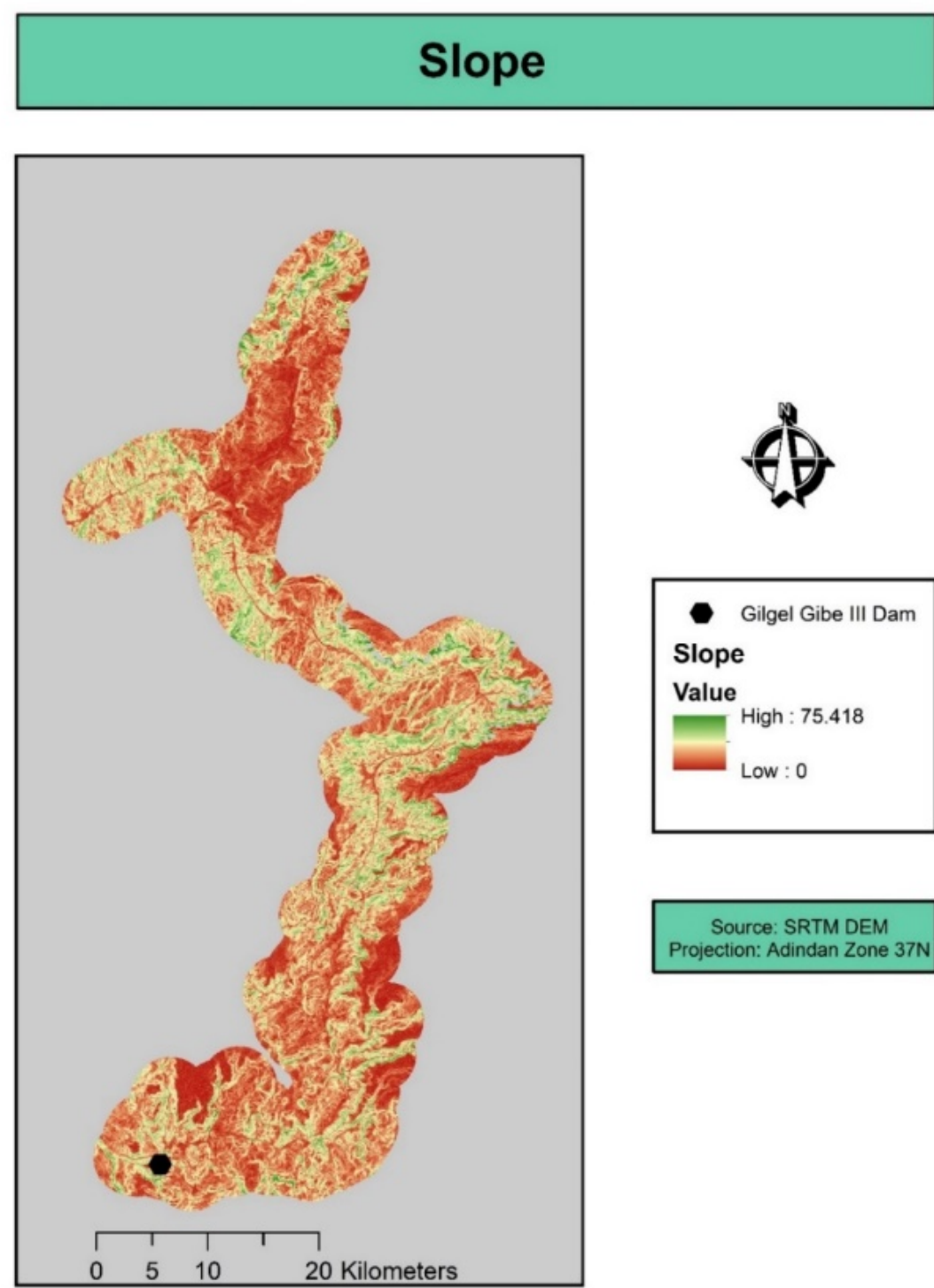

Gilgel Gibe III Dam Slope

Value

High : 75.418

Low : 0

Source: SRTM DEM Projection: Adindan Zone 37N

Figure A1. Slope of Study Area 


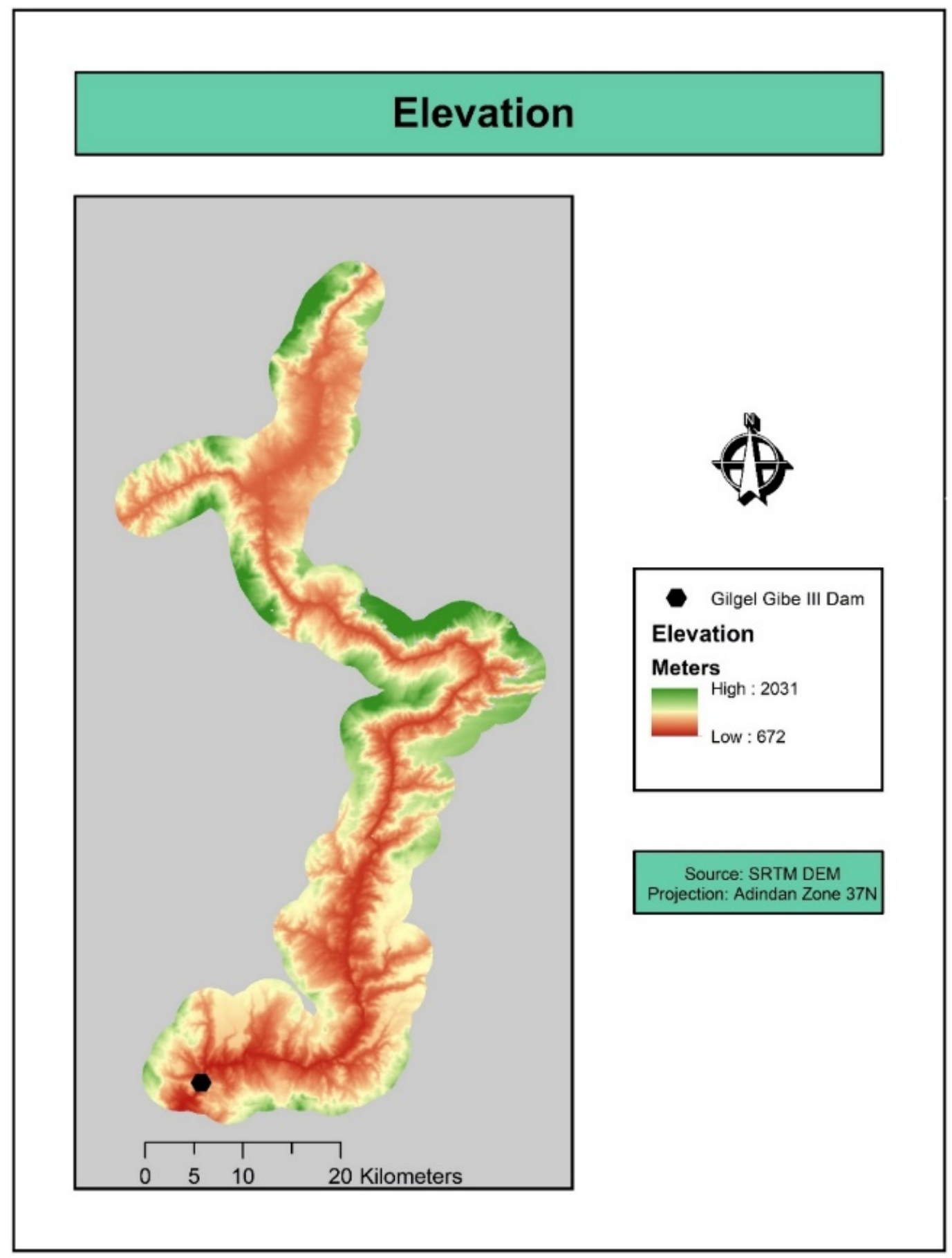

Figure A2. Slope of Study Area 


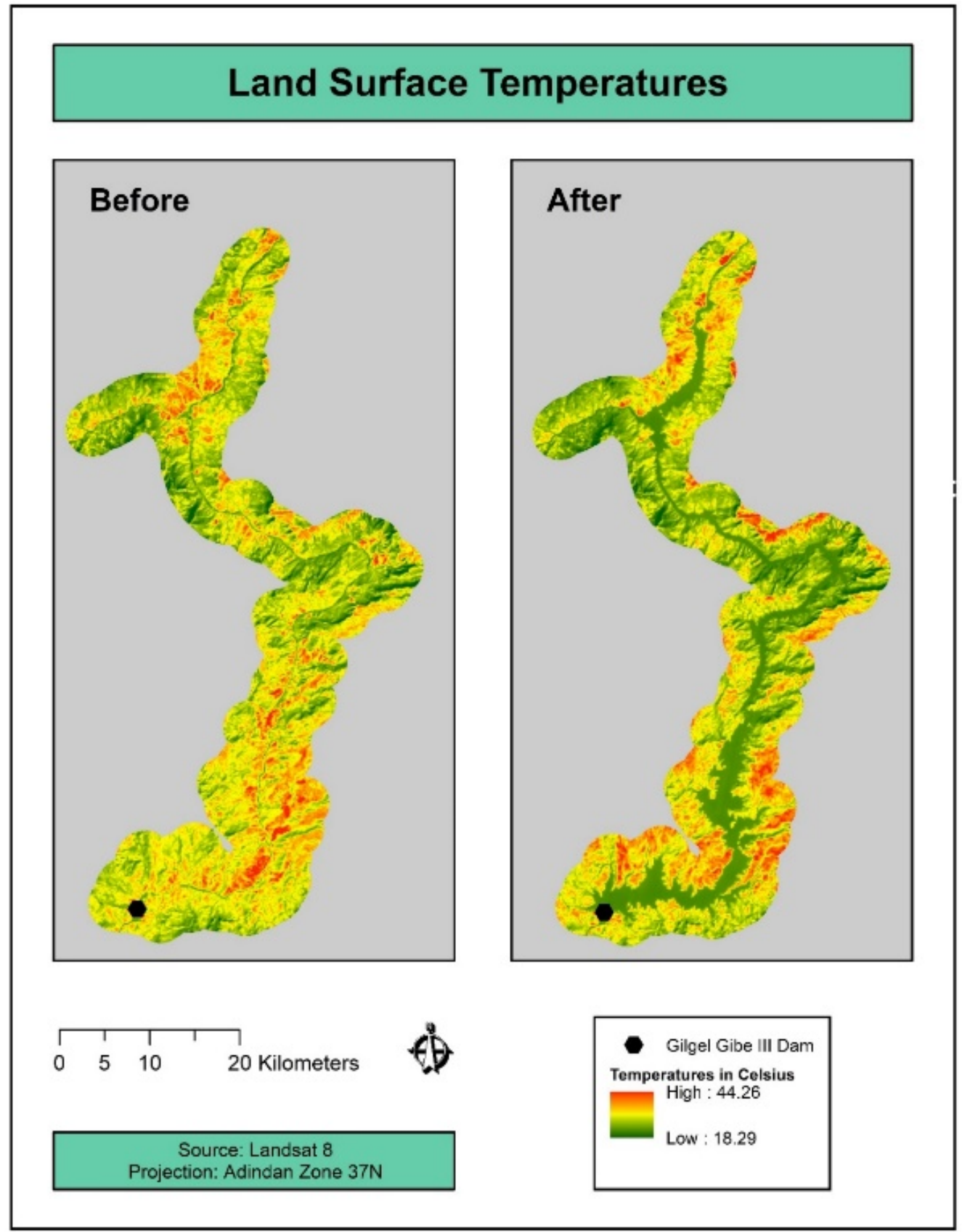

Figure A3. Land Surface Temperatures of Study Area Before and After the Gilgel Gibe III Hydroelectric Dam 


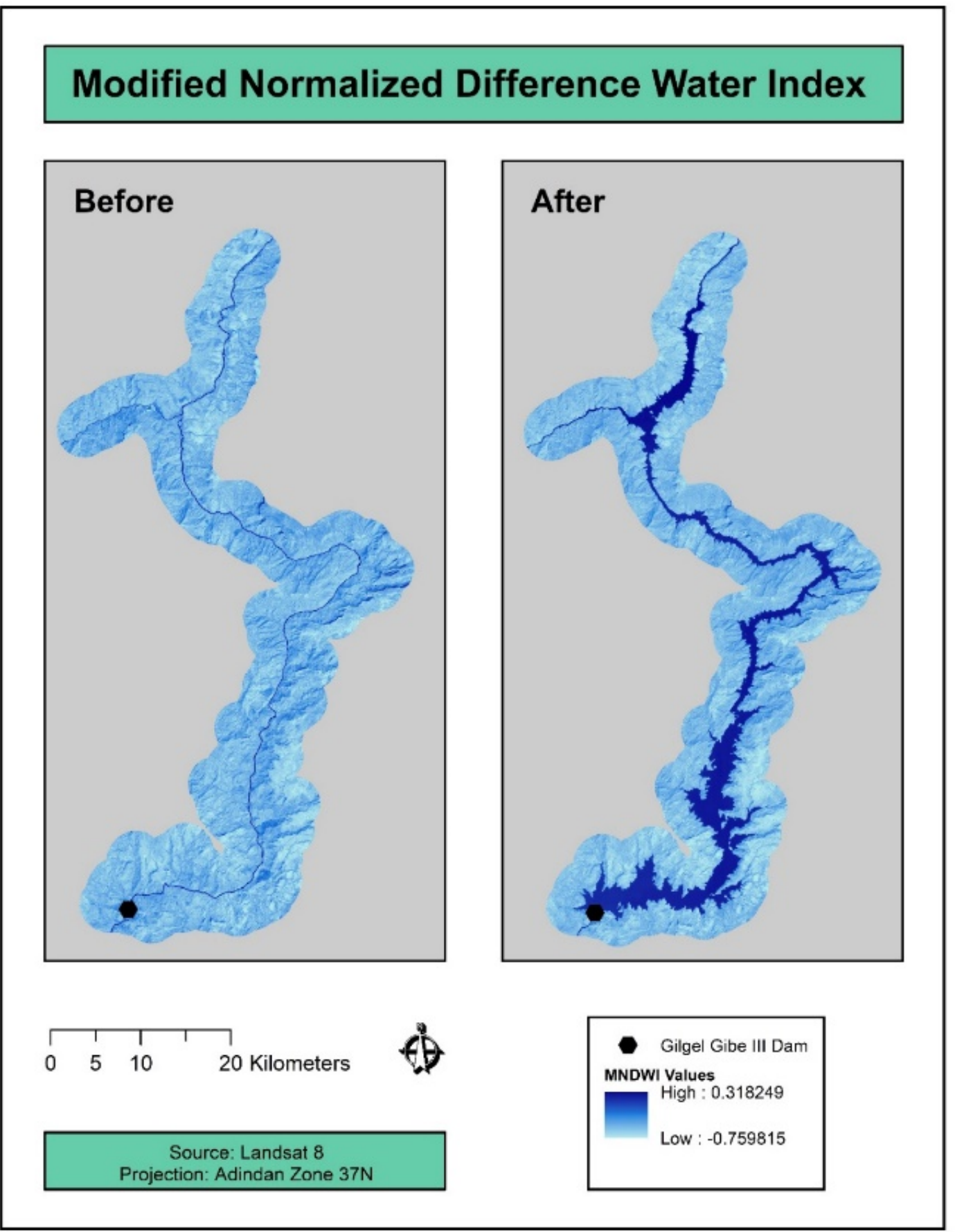

Figure A4. Modified Normalized Difference Water Index Values in the Study Area Before and After the Gilgel Gibe III Hydroelectric Dam 


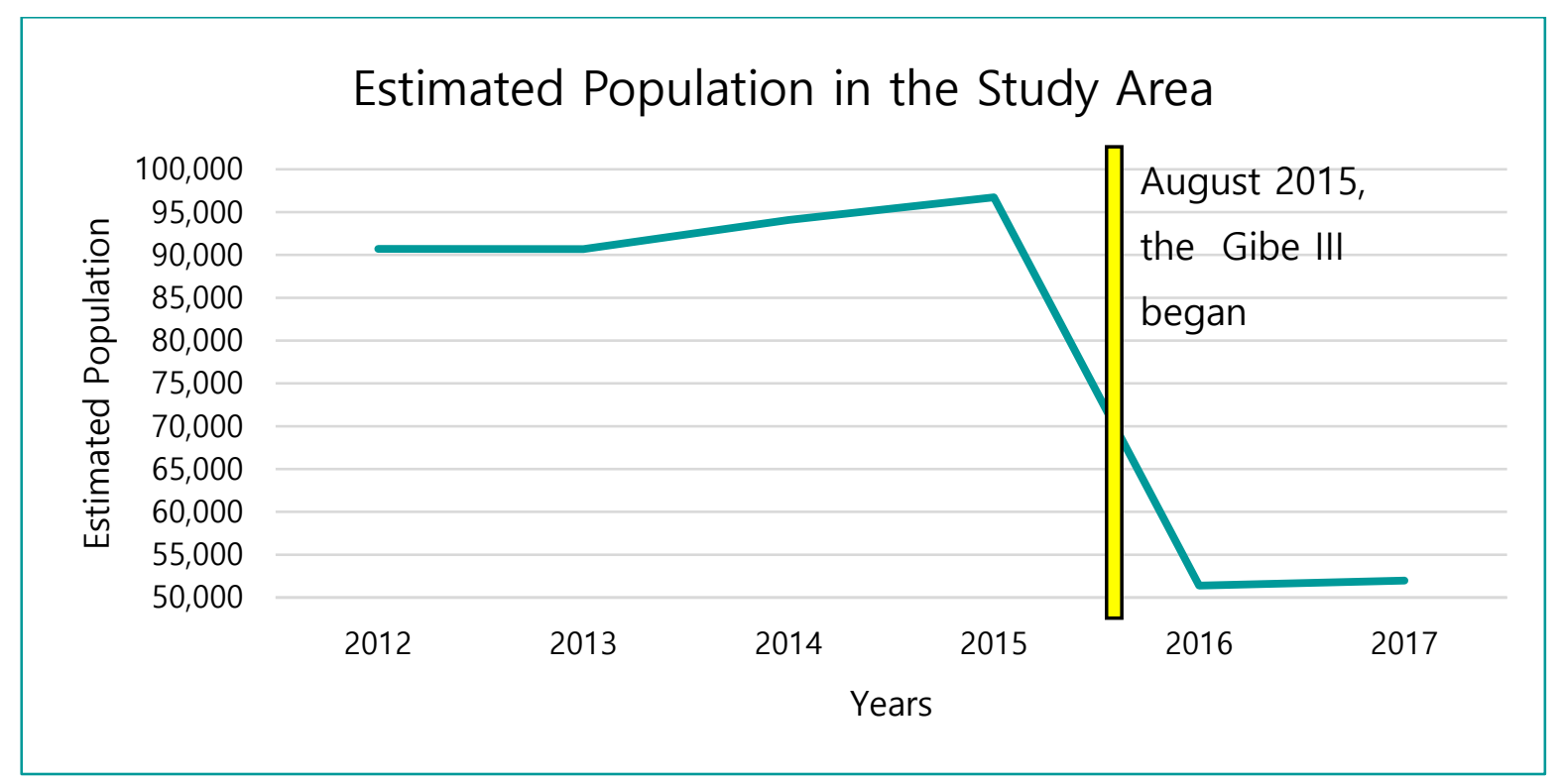

Figure A5. Graph of Yearly Population Estimates within the Study Area

Source: This product was made utilizing the LandScan $(2012,2013,2014,2015,2016,2017)^{\mathrm{TM}}$ High Resolution global Population Data Set copyrighted by UT-Battelle, LLC, operator of Oak Ridge National Laboratory under Contract No. DE-AC05-00OR22725 with the United States Department of Energy. The United States Government has certain rights in this Data Set. Neither UT-BATTELLE, LLC NOR THE UNITED STATES DEPARTMENT OF ENERGY, NOR ANY OF THEIR EMPLOYEES, MAKES ANY WARRANTY, EXPRESS OR IMPLIED, OR ASSUMES ANY LEGAL LIABILITY OR RESPONSIBILITY FOR THE ACCURACY, COMPLETENESS, OR USEFULNESS OF THE DATA SET. 